\title{
Coherent off-axis undulator radiation from short electron bunches
}

\author{
C.P. Neuman* \\ Free-Electron Laser Laboratory, Duke University, Durham, North Carolina 27708 \\ W. S. Graves \\ Brookhaven National Laboratory, Upton, New York 11973-5000 \\ P. G. O'Shea \\ Institute of Plasma Research, Department of Electrical and Computer Engineering, University of Maryland, \\ College Park, Maryland 20742 \\ (Received 29 September 1999; published 10 March 2000)
}

\begin{abstract}
The nature of off-axis undulator radiation is discussed. Of particular interest is coherent off-axis radiation, where the wavelengths of emission are longer than the electron bunch length. We show how this off-axis radiation may be used to measure relative electron bunch lengths. The theory is presented, and calculated spectra are presented in a number of cases of interest.

PACS numbers: 29.27.Fh, 29.17.+w, 41.60.Cr
\end{abstract}

\section{INTRODUCTION}

For decades there has been interest in obtaining coherent radiation from relativistic free electrons. Schwinger discussed the properties of radiation from accelerated electrons in 1949 [1]. In the early 1950's the (unwanted) coherent radiation in a synchrotron was studied theoretically [2]. Also in that decade, the idea existed to make use of coherent mm-wave radiation from bunched electrons [3]. In recent years, different methods have been used to obtain coherent radiation from free electrons [3]. These methods include passing electrons though thin metal foil to produce transition radiation $[4,5]$, generating synchrotron radiation from bending magnets [6], passing electrons near a metal grating to produce Smith-Purcell radiation $[7,8]$, and generating undulator radiation by passing electrons through periodic arrays of magnets $[9,10]$. In this study, we investigate the possibility of obtaining coherent radiation by observing undulator radiation at an angle to the beam axis.

In addition, there is a continuing challenge to accurately characterize short electron bunches. Short bunches are produced by magnetic compression and, in cases of current interest, are generally less than $500 \mu \mathrm{m}$ long. There has been much interest in recent years in producing ultrashort electron bunches for single-pass free-electron lasers (FELs) [11-17] and linear colliders [15,18]. The quality of the electron beam is of utmost importance for these applications, and beam characteristics and their effects on FEL performance have been studied extensively [19]. The performance of magnetic bunch compression

\footnotetext{
*Corresponding author. Present address: Bldg. 725-D, Brookhaven National Laboratory, Upton, NY 11973. Email address: Neuman@bnl.gov
}

is very sensitive to changes in upstream accelerator components. A real-time, noninterrupting bunch length diagnostic is critical for monitoring and controlling this equipment.

Our study shows that it may be possible to use coherent, off-axis undulator radiation to determine the relative length of an electron bunch without intercepting or substantially deflecting the electron beam. Radiation emitted at wavelengths longer than the bunch length will be coherent and thus significantly more intense. Changes in the bunch length may be obtained by measuring changes in the total energy of this off-axis radiation. We show that the total radiated energy increases as the bunch length decreases. Since no microbunching is necessary to obtain coherent radiation, a short undulator may be used, and the electron bunch is passed relatively undisturbed. The result is a nondestructive electron beam diagnostic. This relative bunch length monitor will be used in conjunction with other diagnostic techniques, such as the rf zero-phasing method [20], to determine absolute bunch length.

While this beam diagnostic may be useful for bunches used in short wavelength FELs, it is not itself an FEL. That is, it does not create or amplify coherent light by using the process of microbunching. Therefore, in contrast to a short wavelength FEL, the radiative process in this diagnostic is not sensitive to energy spread. This characteristic makes the diagnostic useful even in cases where the beam quality is not well characterized or is unstable.

Another technique for determining bunch length by measuring coherent radiation is to use bending magnet radiation [21]. The advantages of using an undulator rather than a bending magnet are that it may be placed in a straight section of a linac and that it creates little or no dispersion or emittance growth. This allows for a number of such diagnostic installations on a long linac.

(C) 2000 The American Physical Society

030701-1 


\section{THEORY}

\section{A. Introduction: off-axis undulator radiation}

In most descriptions of undulator radiation, the discussion is limited to small angles of observation, particularly angles less than $1 / \gamma \sqrt{N_{w}}$ radians [22], where $N_{w}$ is the number of periods in the undulator. The small-angle approximation is sometimes even used in cases where the radiation is described as "off-axis" [23]. In this study of off-axis radiation, radiation outside the narrow $1 / \gamma \sqrt{N_{w}}$ cone is explored.

The peak wavelength of undulator radiation increases with angle

$$
\lambda_{0}(\theta)=\frac{\lambda_{w}}{2 \gamma^{2}}\left[\left(1+a_{w}^{2}\right) \cos \theta+2 \gamma^{2}(1-\cos \theta)\right],
$$

where $\theta$ is the angle of observation, $\lambda_{w}$ is the undulator period, $\gamma$ is the electron kinetic energy in units of rest mass, and $a_{w}=e B_{\mathrm{rms}} \lambda_{w} / 2 \pi m c$ is the rms undulator parameter, or dimensionless rms vector potential. Equation (1) is derived in Sec. IIB. The dependence of the fundamental wavelength on the angle of observation is seen in Fig. 1 for typical parameter values $\left(\lambda_{w}=4 \mathrm{~cm}\right.$ and $\left.a_{w}=1\right)$. For $\gamma=400, \lambda_{0}(0)=250 \mathrm{~nm}$ on-axis, and $\lambda_{0}\left(10^{\circ}\right)=600 \mu \mathrm{m}$.

For an infinitely long undulator, we assume that all of the energy is radiated at the peak wavelength. For small angles, this wavelength is much shorter than a bunch length. The electrons radiate incoherently, and the intensity of the radiation scales linearly with the number of electrons. In contrast, at a large enough angle, the peak wavelength may be longer than a bunch length. In this case, the electrons radiate coherently, and the intensity of the radiation scales with the square of the number of electrons. The coherent radiation is more intense than the incoherent radiation by a factor equal to the number of electrons, which is typically on the order of $10^{9}$. This

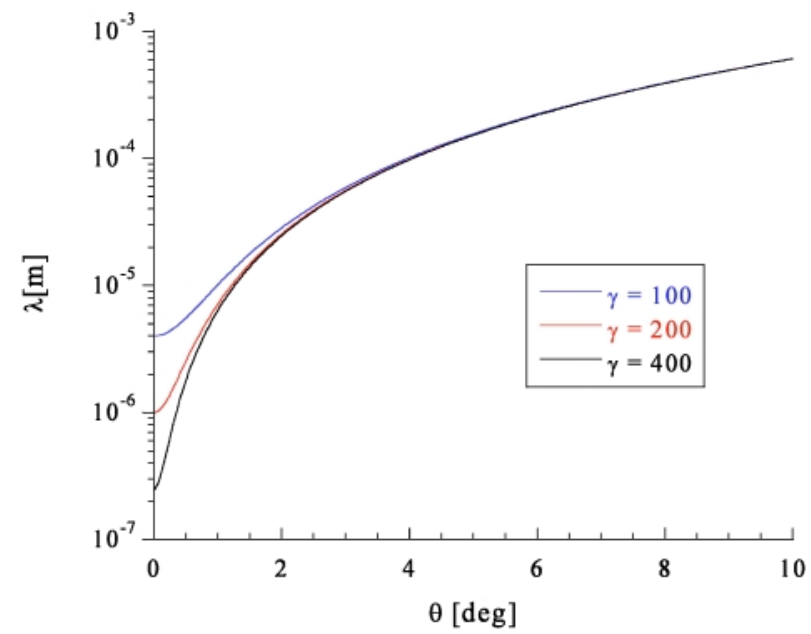

FIG. 1. (Color) Fundamental wavelength as a function of angle of observation for various electron energies. suggests that the bunch length could be measured by observing radiation at various angles and determining the angle at which the radiation becomes coherent. That angle would correspond to a certain wavelength of the radiation, and that wavelength would correspond to a certain bunch length.

However, for a finite length undulator, we cannot assume that all of the radiation is radiated at the peak wavelength. First, the power spectrum of undulator radiation displays the familiar $\operatorname{sinc}^{2}\left[N_{w} \pi\left(\Delta \omega / \omega_{0}\right)\right]$ behavior. While the spectrum may have a narrow peak about the fundamental wavelength, it still includes radiation at other wavelengths. Those wavelengths that are longer than the bunch length will radiate coherently and will thus be significantly more intense, as seen in Fig. 6, where most of the energy of the radiation is seen to be at wavelengths longer than the fundamental. Second, we show below that near-field effects cause the fundamental peak to be shifted and broadened, thus obscuring the meaning of the peak wavelength as defined in Eq. (1).

The fact that radiation is present at frequencies different from, and even far from, the fundamental peak provides for a method of determining bunch length without having to observe various angles, which would be a difficult experimental task. In the method adopted in this study, the radiation at a fixed angle is observed for wavelengths near the length of the electron bunch. Radiation of wavelengths longer than the bunch length will radiate coherently. Thus by observing this off-axis undulator radiation, we may be able to determine the electron bunch length. Of course, the same method could be used for onaxis radiation, but this would require steering the beam from its axis in order to observe the radiation. In that case, the diagnostic would not be noninterrupting as in the off-axis case.

\section{B. Derivation of Eq. (1)}

Equation (1) is derived by examining the electronundulator interaction in both the lab frame and the electron's frame. In the lab frame, an electron moves along the $\hat{z}$ axis with velocity $\bar{\beta}_{z} c$ towards an undulator field

$$
\vec{B}=-B_{0} \sin \left(k_{w} z\right) \hat{y} .
$$

$B_{0}$ is the peak magnetic field of the undulator, $\lambda_{w}$ is the undulator period, and $k_{w}=2 \pi / \lambda_{w}$. In the electron's frame, the undulator field is Lorentz transformed into the fields

$$
\begin{aligned}
\vec{E} & =-\nu \gamma B_{0} \sin \left(\frac{2 \pi}{\lambda^{\prime}} z\right) \hat{x}, \\
\vec{B} & =-\gamma B_{0} \sin \left(\frac{2 \pi}{\lambda^{\prime}} z\right) \hat{y} .
\end{aligned}
$$


The undulator period $\lambda_{w}$ is Lorentz contracted to

$$
\lambda^{\prime}=\frac{\lambda_{w}}{\gamma} .
$$

Since $\vec{E}^{\prime} \perp \vec{B}^{\prime}$ and $E^{\prime} / B^{\prime} \approx c$ for $\gamma \gg 1$, the electron sees the undulator field as a photon. This "virtual photon" scatters off the electron, and assuming negligible recoil, the scattered photon has a wavelength equal to $\lambda^{\prime}$. This wave is Doppler shifted when viewed in the lab frame, according to

$$
\lambda_{0}=\gamma \lambda^{\prime}\left(1-\bar{\beta}_{z} \cos \theta\right),
$$

where $\bar{\beta}_{z} \approx 1-\left[\left(1 / 2 \gamma^{2}\right)\left(1+a_{w}^{2}\right)\right]$. The result, after substitution, is Eq. (1),

$$
\lambda_{0}(\theta)=\frac{\lambda_{w}}{2 \gamma^{2}}\left[\left(1+a_{w}^{2}\right) \cos \theta+2 \gamma^{2}(1-\cos \theta)\right] .
$$

In most publications, the wavelength is described with a small-angle approximation. Equation (1) is valid for all angles.

Note that for $\gamma$ large and $\theta$ greater than a few degrees, Eq. (1) becomes

$$
\lambda_{0}(\theta) \approx \lambda_{w}(1-\cos \theta),
$$

which is independent of the electron beam energy. This behavior is seen in Fig. 1 for $\theta>5^{\circ}$.

\section{Radiated energy calculations for single electron}

We calculate the total energy and the spectral energy of the emitted undulator radiation with a straightforward approach using Liénard-Wiechert potentials [24]. In previous work, the radiated electric field was expressed as a sum of Gaussian modes [25]. That interesting approach had the disadvantage of requiring many modes for an accurate description of the field. Calculations are simpler with the approach used in this study.

The geometry for the calculation is shown in Fig. 2.

The total power per unit solid angle radiated by an accelerating electron, in the electron's time, is given by

$$
\begin{aligned}
\frac{d P\left(t^{\prime}\right)}{d \Omega} & =R^{2} \vec{S} \cdot \hat{n}(1-\hat{n} \cdot \vec{\beta}) \\
& =c \varepsilon_{0} E^{2} R^{2}(1-\hat{n} \cdot \vec{\beta}),
\end{aligned}
$$

where $\vec{S}$ is the Poynting vector. The total energy per unit solid angle is obtained by integrating over time. The electric field $\vec{E}$ can be derived from Liénard-Wiechert potentials,

$$
\begin{aligned}
\vec{E}=\frac{q^{2}}{4 \pi \varepsilon_{0}} & \left\{\frac{1}{\gamma^{2} R^{2}} \frac{\hat{n}-\vec{\beta}}{(1-\hat{n} \cdot \vec{\beta})^{3}}\right. \\
& \left.+\frac{1}{c R} \frac{\hat{n} \times[(\hat{n}-\vec{\beta}) \times \dot{\vec{\beta}}]}{(1-\hat{n} \cdot \vec{\beta})^{3}}\right\} .
\end{aligned}
$$

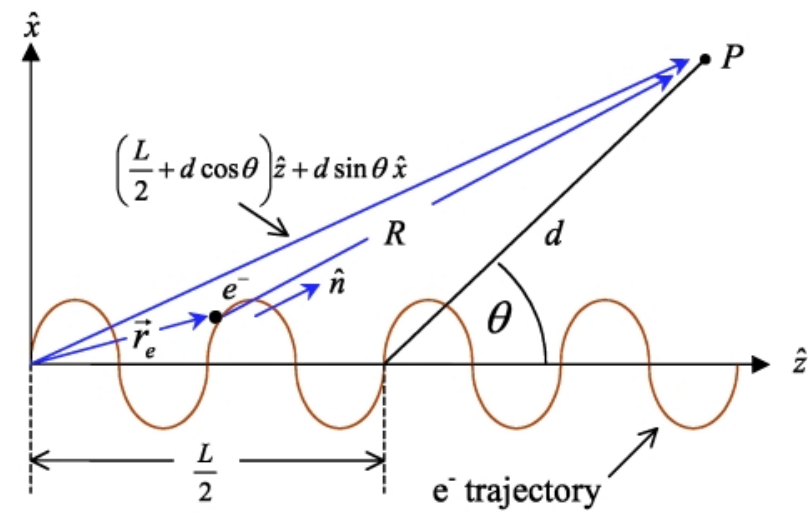

FIG. 2. (Color) Geometry for energy calculations. $P$ is the point of observation, $\vec{r}_{e}$ is the instantaneous position of the electron, $R$ and $\hat{n}$ are the distance and direction, respectively, from the electron to the point of observation, and $d$ is the distance from the center of the undulator to the point of observation.

The first term represents the velocity field, which does not radiate and is thus disregarded in this study. The second term represents the radiating acceleration field and depends on both the velocity and acceleration of the electron. Calculations for the cases in this study have been compared to those performed with the code SRW [26] which includes the velocity term. The comparison verifies that the velocity field term is negligible in the cases considered in this study.

The energy per unit solid angle is thus

$$
\begin{aligned}
\frac{d W}{d \Omega}= & \frac{1}{4 \pi \varepsilon_{0}} \frac{q^{2}}{4 \pi c} \\
& \times \int_{0}^{L / \bar{\beta}_{z} c} \frac{|\hat{n} \times[(\hat{n}-\vec{\beta}) \times \dot{\vec{\beta}}]|^{2}}{(1-\hat{n} \cdot \vec{\beta})^{5}} d t .
\end{aligned}
$$

$L$ is the undulator length, and $\bar{\beta}_{z}$ is the normalized average longitudinal velocity of the electron. Thus, $L / \bar{\beta}_{z} c$ is the time that the electron leaves the undulator.

Since the energy per unit solid angle in the observer's time may be expressed as a frequency integral,

$$
\frac{d W}{d \Omega}=\int_{-\infty}^{\infty}|\vec{A}(t)|^{2} d t=\int_{-\infty}^{\infty}|\vec{A}(\omega)|^{2} d \omega
$$

where $\vec{A}(\omega)$ is the Fourier transform of $\vec{A}(t)$, and $\vec{A}(t)$ is defined as

$$
\vec{A}(t) \equiv \sqrt{\varepsilon_{0} c} R \vec{E},
$$

the energy radiated per unit solid angle per unit angular frequency is defined as follows:

$$
\frac{d W}{d \Omega} \equiv \int_{0}^{\infty} \frac{d^{2} I}{d \omega d \Omega} d \omega=\int_{0}^{\infty} 2|\vec{A}(\omega)|^{2} d \omega
$$


This spectral energy is thus given by

$$
\frac{d^{2} I}{d \omega d \Omega}=\frac{\varepsilon_{0}}{c \pi} \frac{q^{2}}{\left(4 \pi \varepsilon_{0}\right)^{2}}\left|\int_{0}^{L / \bar{\beta}_{z} c} e^{i \omega[t+(R / c)]} \frac{\hat{n} \times[(\hat{n}-\vec{\beta}) \times \dot{\vec{\beta}}]}{(1-\hat{n} \cdot \vec{\beta})^{2}} d t\right|^{2} .
$$

The total energy and spectral energy are related to the respective energies per unit solid angle by

$$
\begin{aligned}
W & =\frac{1}{d^{2}}\left(\frac{d W}{d \Omega}\right) \Delta A, \\
\frac{d W}{d \omega} & =\frac{1}{d^{2}}\left(\frac{d^{2} I}{d \omega d \Omega}\right) \Delta A,
\end{aligned}
$$

where $d$ is the distance of the detector from the center of the undulator and $\Delta A$ is the area of the detector entrance aperture.

To evaluate Eqs. (4) and (5), the normalized acceleration and velocity of the electron are needed, along with the approximation $z \approx \bar{\beta}_{z}$ ct. The velocity and acceleration are calculated by applying the Lorentz force equation to the periodic undulator field, Eq. (2), yielding the following well-known expressions:

$$
\begin{aligned}
\dot{\beta}_{x} & =\frac{-a_{w} \sqrt{2}}{\gamma} \sin \left(k_{w} z\right) k_{w} \bar{\beta}_{z} c \\
\dot{\beta}_{z} & =\frac{a_{w}^{2}}{\gamma^{2}} \sin \left(2 k_{w} z\right) k_{w} \bar{\beta}_{z} c \\
\beta_{x} & =\frac{a_{w} \sqrt{2}}{\gamma} \cos \left(k_{w} z\right) \\
\beta_{z} & =\bar{\beta}_{z}-\frac{a_{w}^{2}}{2 \gamma^{2}} \cos \left(2 k_{w} z\right), \\
\bar{\beta}_{z} & \equiv 1-\frac{1}{2 \gamma^{2}}\left(1+a_{w}^{2}\right) .
\end{aligned}
$$

\section{Radiated energy calculations for a bunch of $N_{e}$ electrons}

To include the effects of a bunch of $N_{e}$ electrons, consider the component of the electric field seen at a detector from the $k$ th electron in a bunch (see Fig. 3) [27],

$$
E_{k}(\omega)=E_{0}(\omega) e^{i(\omega / c) \hat{n}_{k} \cdot \vec{r}_{k}}
$$

The total energy is given by

$$
\begin{aligned}
W_{\text {tot }}(\omega) & =W_{0}(\omega)\left|\sum_{k=1}^{N_{e}} e^{i(\omega / c) \hat{n}_{k} \cdot \vec{r}_{k}}\right|^{2} \\
& =W_{0}(\omega) \sum_{k=1}^{N_{e}} e^{i(\omega / c) \hat{n}_{k} \cdot \vec{r}_{k}} \sum_{j=1}^{N_{e}} e^{-i(\omega / c) \hat{n}_{j} \cdot \vec{r}_{j}} \\
& =W_{0}\left(N_{e}+\sum_{j \neq k}^{N_{e}} e^{i(\omega / c)\left(\hat{n}_{k} \cdot \vec{r}_{k}-\hat{n}_{j} \cdot \vec{r}_{j}\right)}\right) .
\end{aligned}
$$

The double sum can be expressed as

$$
\begin{aligned}
& \sum_{j \neq k}^{N_{e}} e^{i(\omega / c)\left[\hat{n}_{k} \cdot \vec{r}_{k}-\hat{n}_{j} \cdot \vec{r}_{j}\right]} \\
& \quad=N_{e}\left(N_{e}-1\right)\left|\int d \vec{r} S(\vec{r}) e^{i(\omega / c) \hat{n} \cdot \vec{r}}\right|^{2},
\end{aligned}
$$

where $S(\vec{r})$ is the normalized electron bunch density function and $N_{e} S(\vec{r})$ is the electron number density function. The result is

$$
W_{N_{e} \text { electrons }}=W_{1 \text { electron }}\left[N_{e}+N_{e}\left(N_{e}-1\right) f(\omega)\right],
$$

where $f(\omega)$ is the bunch form factor

$$
\begin{aligned}
f(\omega)= & \mid \iint d y d z S_{y}(y) S_{z}(z) \\
& \times\left. e^{-i(\omega / c) y \sin \theta+i(\omega / c) z \cos \theta}\right|^{2} .
\end{aligned}
$$

$S_{y}(y)$ and $S_{z}(z)$ are the transverse and longitudinal electron bunch densities, respectively. For a Gaussianshaped bunch,

$$
S_{z}(z)=\frac{e^{-(1 / 2)\left(z / \sigma_{z}\right)^{2}}}{\sqrt{2 \pi} \sigma_{z}}, \quad S_{y}(y)=\frac{e^{-(1 / 2)\left(y / \sigma_{y}\right)^{2}}}{\sqrt{2 \pi} \sigma_{y}},
$$

and

$$
f(\omega)=e^{-\left(k \cos \theta \sigma_{z}\right)^{2}} e^{-\left(k \sin \theta \sigma_{y}\right)^{2}} .
$$

For a top-hat-shaped bunch,

$$
\begin{gathered}
S_{z}(z)=\frac{1}{2 \sqrt{3} \sigma_{z}} \quad \text { for }|z|<\sqrt{3} \sigma_{z}, \\
S_{y}(y)=\frac{1}{2 \sqrt{3} \sigma_{y}} \quad \text { for }|y|<\sqrt{3} \sigma_{y},
\end{gathered}
$$

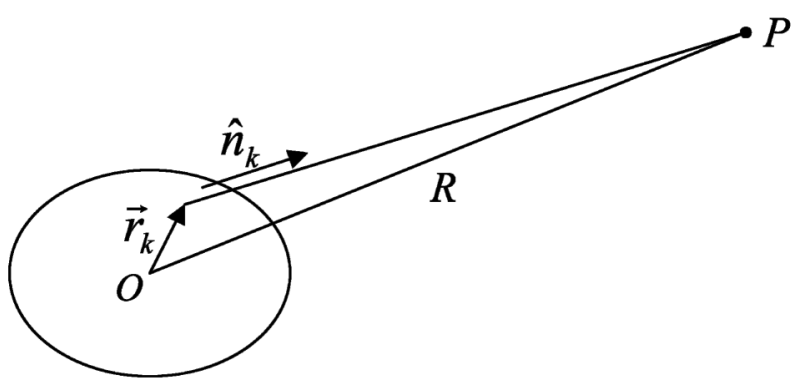

electron bunch

FIG. 3. Geometry for bunch form factor calculation. $P$ is the point of observation, $\vec{r}_{k}$ is the vector from the origin to the $k$ th electron in the bunch, $\hat{n}_{k}$ is the unit vector pointing from the $k$ th particle to the point of observation, and $R$ is the distance from the origin to the point of observation. 
and

$$
f(\omega)=\left(\frac{\sin \left(k \cos \theta \sqrt{3} \sigma_{z}\right)}{k \cos \theta \sqrt{3} \sigma_{z}}\right)^{2}\left(\frac{\sin \left(k \sin \theta \sqrt{3} \sigma_{y}\right)}{k \sin \theta \sqrt{3} \sigma_{y}}\right)^{2} .
$$

In this study we have assumed Gaussian-shaped bunch density functions, except where noted.

Equations (9) and (10) include the coherence effects of the radiation. For short wavelengths $\omega$ is large, $f(\omega) \approx$ 0 , and $W \propto N_{e}$. Thus the energy scales linearly with the number of electrons, as expected for incoherent radiation. For long wavelengths $\omega$ is small, $f(\omega) \approx 1$, and $W \propto$ $N_{e}^{2}$. In this case, the energy scales quadratically with the number of electrons, as expected for coherent radiation. In general, both coherent and incoherent radiation are present at the same time.

The form factor depends on both the length and the width of the electron bunch. However, note that for the range of angles in this study, the form factor depends weakly on the width. This can be seen in Eqs. (11) and (12), above, where the factors relating to the transverse distribution are close to unity. As a result of this weak dependence on the bunch width, only the effects of changes in the bunch length are considered. Furthermore, the widths used in this study, around $100 \mu \mathrm{m}$, are easily measured, but a bunch length of $100 \mu \mathrm{m}$ is not as straightforward to measure. Thus, the bunch length is the quantity of greater interest.

The form factor also depends on the wavelength $\lambda$ through $\omega=(2 \pi / \lambda) c$. Thus, if Eq. (9) were applied to the total energy as given in Eq. (4), an assumption would be made that the radiation consists of only one wavelength. When the spectrum is calculated with Eq. (5), however, Eq. (9) is applied to each frequency, so no such approximation is made. As stated above, for undulator radiation from a finite-length undulator we cannot assume that all the radiation is radiated at the peak wavelength, thus a calculation of the total energy using Eq. (4) is valid only for the single electron case. For an accurate calculation of the total energy for a bunch of electrons, the spectrum must be calculated using Eq. (5), and Eq. (9) must be applied to each frequency calculated. The total energy is then calculated by summing over a specified frequency range.

\section{E. Near-field effects}

Near-field effects are introduced by accounting for the fact that $\hat{n}$ and $R$ change as the electron passes through the undulator (see Fig. 2). Note that the expression "nearfield" here does not refer to the velocity field in Eq. (3), which is sometimes called the "near-field term" in the literature. The vector $R \hat{n}$ can be expressed as

$$
R(t) \hat{n}(t)=\left(\frac{L}{2}+d \cos \theta\right) \hat{z}+d \sin \theta \hat{x}-\vec{r}_{e}(t),
$$

where $\vec{r}_{e}(t)$ is the electron trajectory. $\vec{r}_{e}$ is calculated by integrating Eqs. (8) with the approximation $z \approx \bar{\beta}_{z} c t$,

$$
\begin{aligned}
& r_{x}=\frac{a_{w} \sqrt{2}}{\gamma k_{w} \bar{\beta}_{z}} \sin \left(k_{w} \bar{\beta}_{z} c t\right), \\
& r_{z}=\bar{\beta}_{z} c t-\frac{a_{w}^{2}}{4 \gamma^{2} k_{w} \bar{\beta}_{z}} \sin \left(2 k_{w} \bar{\beta}_{z} c t\right) .
\end{aligned}
$$

Thus, $R(t)$ is obtained by taking the magnitude of Eq. (13), and $\hat{n}(t)$ is obtained by dividing Eq. (13) by $R(t)$.

\section{CALCUlations}

Equation (5) is evaluated numerically to produce the following results, which offer insight into the nature of off-axis undulator radiation as well as its use for a beam diagnostic.

\section{A. Off-axis spectra}

The spectra for different observation angles are presented in Fig. 4. Notice that the even harmonics appear only at nonzero angles, as expected for undulator radiation. For on-axis radiation, the spectrum displays the expected $\operatorname{sinc}^{2}\left[N_{w} \pi\left(\Delta \omega / \omega_{0}\right)\right]$ behavior. For off-axis radiation, this behavior is modified slightly.

\section{B. Near-field effects}

Calculations show that near-field effects have a significant effect on the energy spectrum for detector distances of less than $1 \mathrm{~m}$ (see Fig. 5). The fundamental peak is shifted to higher frequencies and is broadened. Without near-field effects, the spectrum displays the expected $\operatorname{sinc}^{2}\left[N_{w} \pi\left(\Delta \omega / \omega_{0}\right)\right]$ behavior on-axis, and slight variations of this behavior off-axis, as seen in Fig. 4. This behavior is disturbed in the near-field calculation. As the detector distance becomes large, greater than $1 \mathrm{~m}$ in this case, the near-field and far-field calculations begin to have similar behavior as expected. In addition, for $\theta=0^{\circ}$, the near-field effects disappear since $\hat{n}$ does not change and $R$ is not present in the calculation.

\section{Energy spectra}

Plots of energy spectra are seen in Figs. 6 and 7 for a Gaussian and top hat bunch distribution, respectively. Each plot shows the single-electron spectrum as well as the spectrum for a bunch of electrons. The jump in intensity corresponding to coherent radiation is clearly seen for wavelengths approximately greater than the bunch length. Note that most of the energy is radiated at wavelengths different from the fundamental wavelength.

It is clearly seen that the spectrum from the top-hatshaped bunch contains more high-frequency components than the spectrum from the Gaussian-shaped bunch. This is expected since the sharp features of the top hat distribution cause some coherent radiation at short wavelengths. 

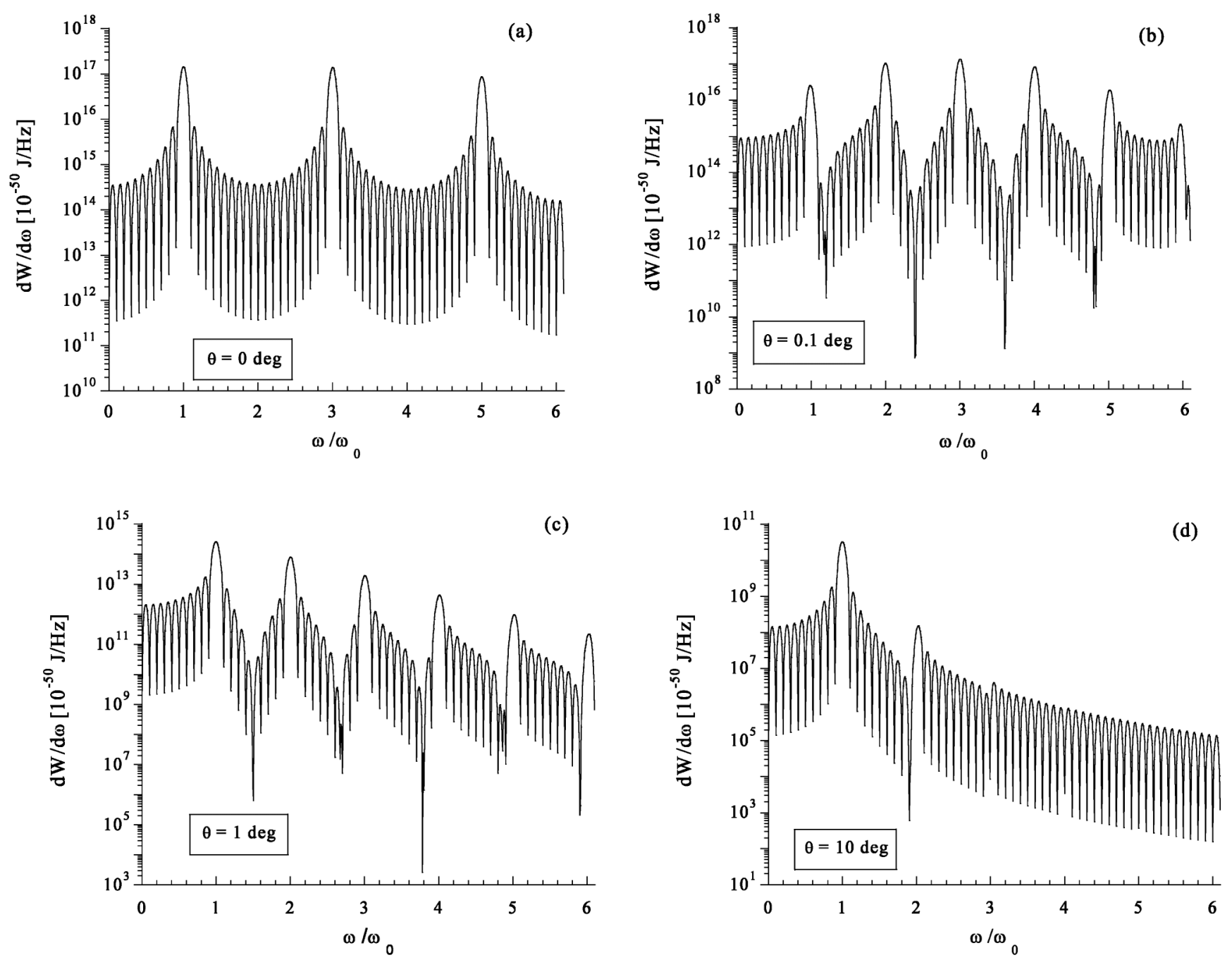

FIG. 4. Spectra for different observation angles. $\lambda_{w}=4 \mathrm{~cm}, N_{w}=10, a_{w}=1, \gamma=400$. (a) $\theta=0^{\circ}$, (b) $\theta=0.1^{\circ}$, (c) $\theta=1^{\circ}$, and (d) $\theta=10^{\circ}$.

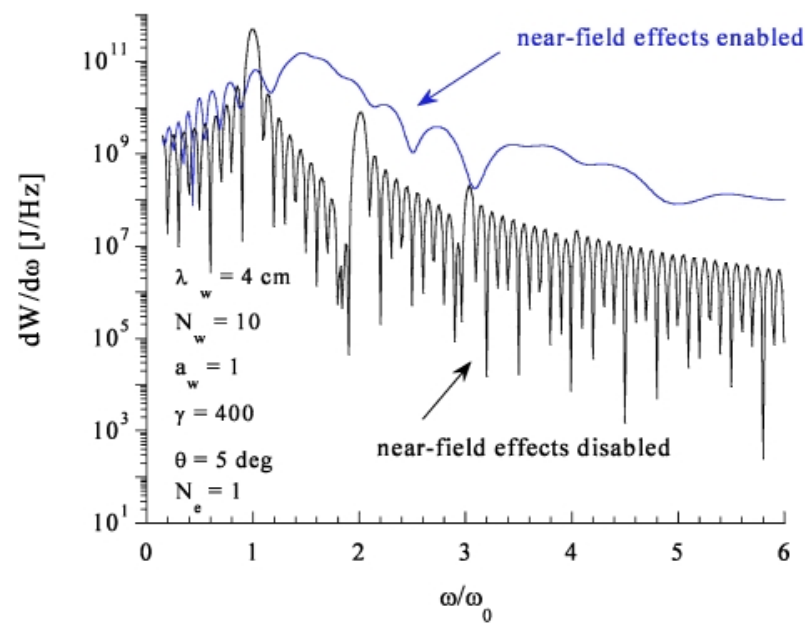

FIG. 5.(Color) Effects of near-field effects on the energy spectrum for a single electron. The detector distance is $0.5 \mathrm{~m}$.

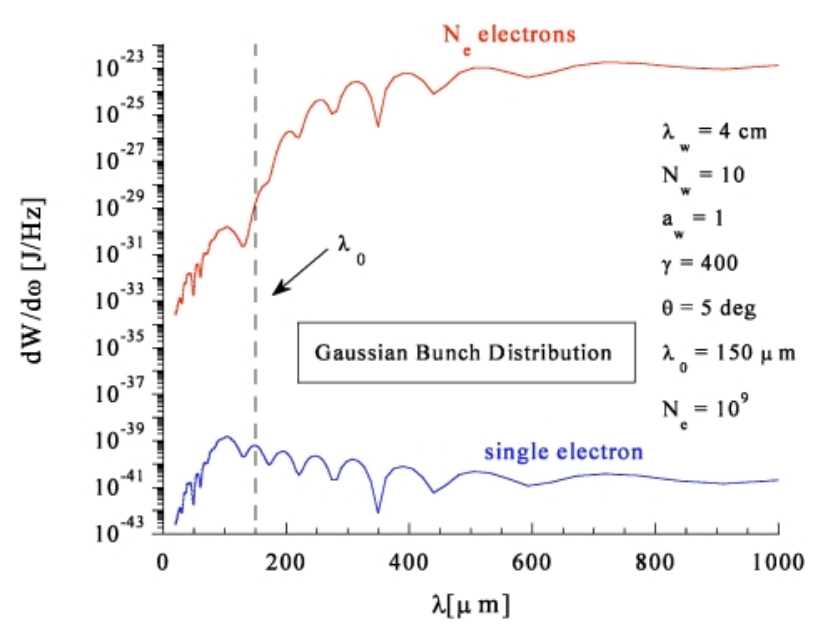

FIG. 6. (Color) Energy spectrum for a Gaussian bunch distribution. The electron bunch has an rms length $\sigma_{z}=100 \mu \mathrm{m}$. 


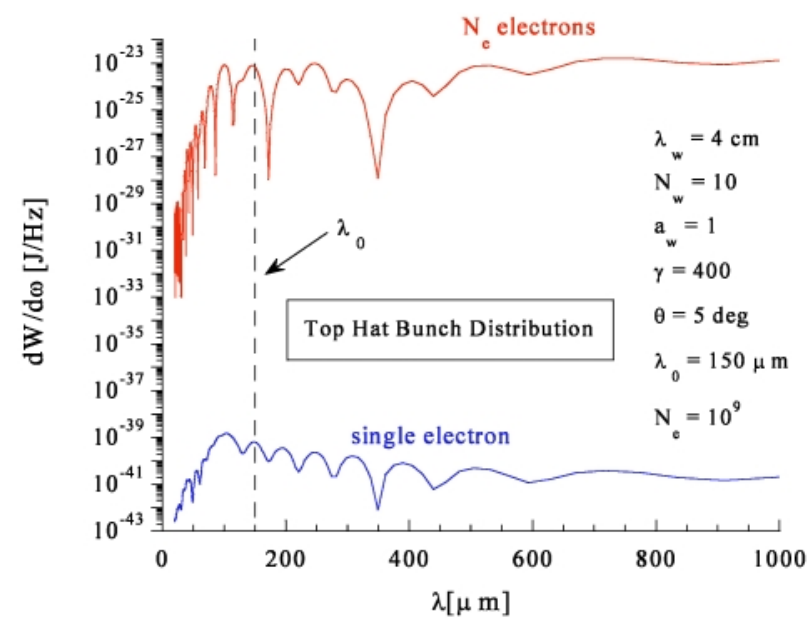

FIG. 7. (Color) Energy spectrum for a top hat bunch distribution. The electron bunch has an rms length $\sigma_{z}=100 \mu \mathrm{m}$.

\section{Energy spectra for various bunch lengths}

The energy spectra show the expected jump in intensity due to coherent radiation of wavelengths greater than the bunch length. This behavior is seen most clearly in the calculation which assumes a Gaussian bunch shape. In the case of a top hat bunch shape there is more radiation at short wavelengths, and the spectrum has more features, making it difficult to distinguish between the spectra of different bunch lengths. In practice, our bunches are not expected to be Gaussian in shape. Bunches may exhibit features such as an abrupt leading edge and a long tail which would produce a spectrum with a complicated structure, as in the case of top-hat-shaped bunches. Thus, it may be difficult to determine bunch length simply by observing spectra. However, the total integrated energy changes with bunch shape, as shown

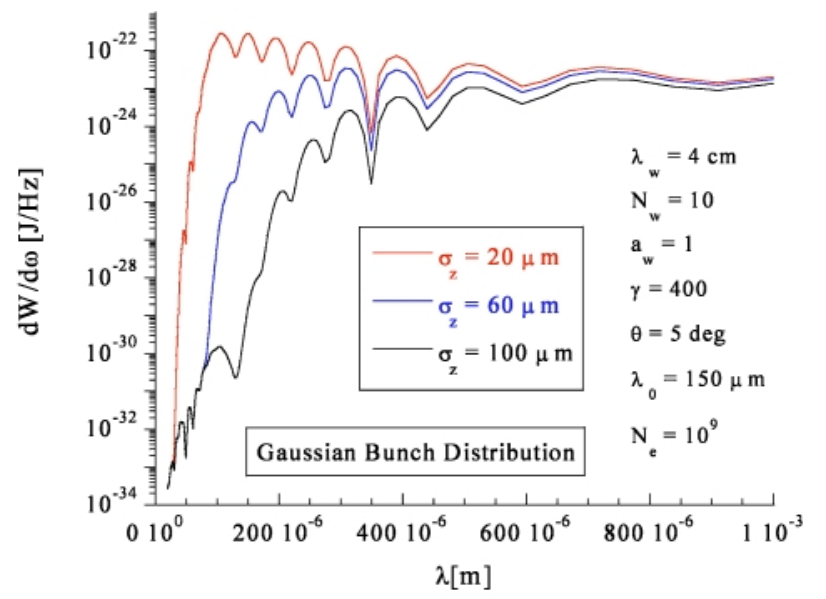

FIG. 8. (Color) Energy spectra for different bunch lengths of a Gaussian-shaped electron beam.

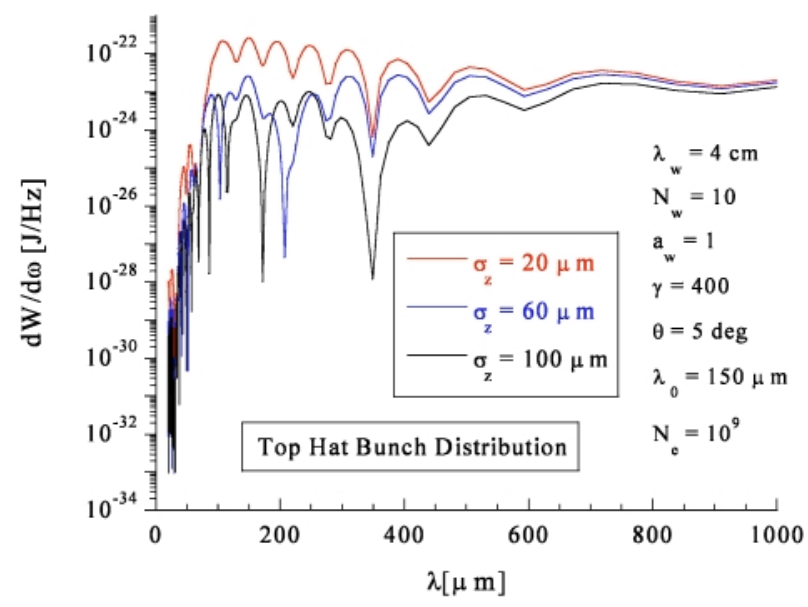

FIG. 9. (Color) Energy spectra for different bunch lengths of a top-hat-shaped electron beam.

below, and provides a more robust diagnostic to measure relative bunch lengths.

\section{E. Total integrated energy}

In Figs. 8 and 9, the total integrated energy can be seen to decrease as the bunch length increases. Figure 10 shows this behavior quantitatively. A detector will detect the total energy, which changes by as much as 3 orders of magnitude when the bunch length increases from 20 to $120 \mu \mathrm{m}$. Thus, monitoring the total energy would be a useful way to determine changes in the bunch length. As the conditions or parameters of an accelerator are changed, the detected energy of off-axis undulator radiation changes and indicates changes in the bunch length. Thus, the bunch length may be optimized while parameters are being changed.

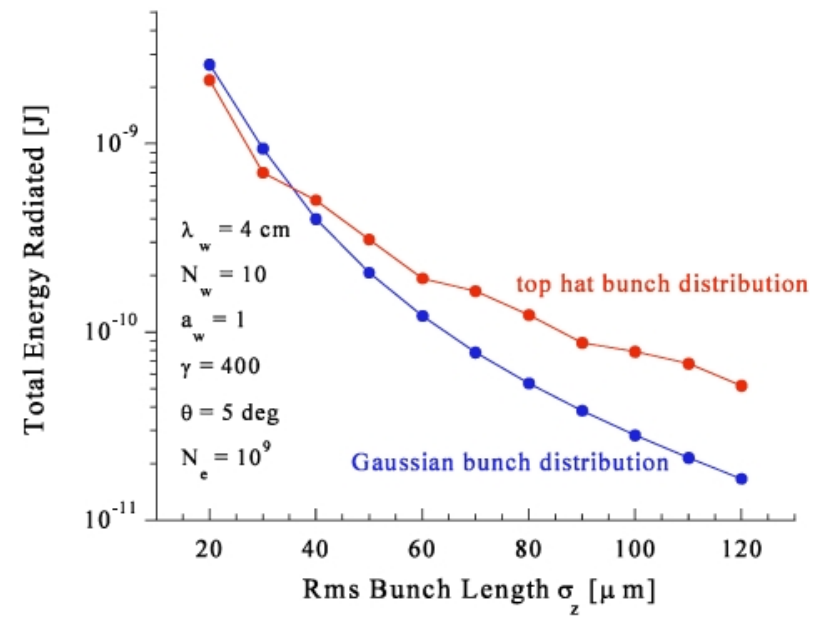

FIG. 10. (Color) Total integrated energy for various rms bunch lengths. The integration is performed from $20 \mu \mathrm{m}$ to $1 \mathrm{~mm}$, which is the anticipated response of the detector. 
TABLE I. Electron beam parameters.

\begin{tabular}{lc}
\hline \hline Electron beam energy & $210 \mathrm{MeV}$ \\
Bunch charge & $0.5 \mathrm{nC}$ \\
Bunch width & $100 \mu \mathrm{m}$ \\
Bunch length & $20-500 \mu \mathrm{m}$ \\
rms normalized emittance & $5 \mu \mathrm{m}$ \\
\hline \hline
\end{tabular}

\section{EXPERIMENT}

The goals of the experiment are to observe and characterize coherent off-axis undulator radiation and to use it to measure relative bunch lengths.

The experiment is planned for the DUV-FEL facility, a new priority for BNL designed to produce a coherent light source in the ultraviolet range [28]. The linear accelerator will produce $210 \mathrm{MeV}$ electrons. Electron beam parameters are given in Table I. The undulator for the experiment is a 12-period prototype of the $10 \mathrm{~m}$ NISUS undulator [29], now installed at the DUV-FEL. See Table II for undulator parameters.

The liquid-helium-cooled bolometer which will be used to detect the off-axis radiation has a noise floor of $1 \mathrm{pJ}$. This energy is an order of magnitude lower than the lowest calculated energy shown in Fig. 11, where the parameters are similar to those in Tables I and II. Thus, such a detector should suffice for this experiment. An FTIR Michelson interferometer spectrometer will be used to determine the spectral content of the radiation. Radiation will exit the beam line through an aperture with a $2 \mathrm{~cm}$ diameter diamond window, after being reflected away from the beam line by an $11 \mathrm{~mm}$ diameter mirror (see Fig. 11). The angular aperture of the system is $13 \mathrm{mrad}$.

\section{CONCLUSION}

We have explored the nature of off-axis undulator radiation and have shown that the radiation is coherent for wavelengths greater than the bunch length. By observing the total energy of off-axis undulator radiation, we will be able to detect changes in the bunch length. Thus, offaxis undulator radiation may be used as a noninterrupting electron beam diagnostic. Such a diagnostic would be especially useful in single-pass high-gain FELs, where the length of a short electron bunch is critical. Relative bunch lengths could be observed in real time, and the FEL output could be optimized while the FEL is operating. An experiment to observe coherent off-axis undulator radiation and to test its use in measuring electron bunches is planned for the DUV-FEL facility at BNL.

TABLE II. Undulator parameters for NISUS undulator.

\begin{tabular}{lc}
\hline \hline Undulator period & $3.89 \mathrm{~cm}$ \\
Number of periods & 12 \\
rms undulator parameter $\left(a_{w}\right)$ & 1.4 \\
\hline \hline
\end{tabular}

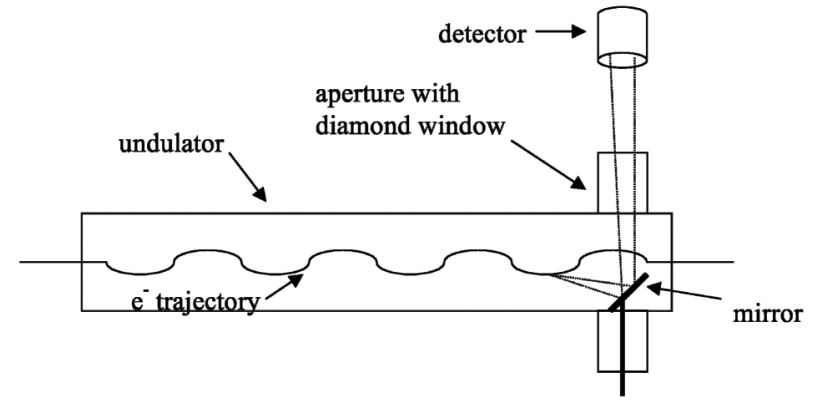

FIG. 11. Off-axis radiation experiment.

\section{ACKNOWLEDGMENTS}

The authors wish to thank Jim Murphy of BNL for helpful discussions on electromagnetic theory, Larry Carr and Gwyn Williams of BNL for helpful discussions on detection, and John Madey of the University of Hawaii for early discussions on the nature of the radiation. Computational support, in the form of Cray computer time, was provided by the North Carolina Supercomputing Center. This work was supported in part by a U.S. Army AASERT grant to the Duke University FEL Lab.

[1] J. Schwinger, Phys. Rev. 75, 1912 (1949).

[2] J. Nodvick and D. Saxon, Phys. Rev. 96, 180 (1954).

[3] G. P. Gallerano, A. Doria, E. Giovenale, and G. Messina, Nucl. Instrum. Methods Phys. Res., Sect. A 358, 78 (1995).

[4] J.-C. Denard, D. Rule, R. Fiorito, P. Adderley, K. Jordan, and K. Capek, in Beam Instrumentation Workshop, edited by G. H. Mackenzie, B. Rawnsley, and J. Thompson, AIP Conf. Proc. No. 333 (AIP, New York, 1996), p. 224.

[5] E. Nishimura, Nucl. Instrum. Methods Phys. Res., Sect. A 358, 89 (1995).

[6] T. Nakazato, Phys. Rev. Lett. 63, 1245 (1989).

[7] Y. Shibata, S. Hasebe, K. Ishi, S. Ono, M. Ikezawa, T. Nakazato, M. Oyamada, S. Urasawa, T. Takahashi, T. Matsuyama, K. Kobayashi, and Y. Fujita, Phys. Rev. E 57, 1061 (1998).

[8] J. H. Brownell, J. Walsh, H. G. Kirk, R. C. Fernow, and S. H. Robertson, Nucl. Instrum. Methods Phys. Res., Sect. A 393, 323 (1997).

[9] H. Motz, J. Appl. Phys. 22, 527 (1951).

[10] J. M. J. Madey, J. Appl. Phys. 42, 1906 (1971).

[11] K.-J. Kim and M. Xie, Nucl. Instrum. Methods Phys. Res., Sect. A 331, 359 (1993).

[12] K.-J. Kim, Nucl. Instrum. Methods Phys. Res., Sect. A 358, 31 (1995).

[13] R. Bonifacio, C. Pellegrini, and L. M. Narducci, Opt. Commun. 50, 373 (1984).

[14] L.-H. Yu, Phys. Rev. A 44, 5178 (1991).

[15] C. Pellegrini, in Micro Bunches Workshop, edited by E. B. Blum, M. Dienes, and J.B. Murphy, AIP Conf. Proc. No. 367 (AIP, New York, 1996), p. 3.

[16] P. G. O'Shea, Nucl. Instrum. Methods Phys. Res., Sect. A 358, 36 (1995). 
[17] P. G. O'Shea, C.P. Neuman, J.M. J. Madey, and H. P. Freund, Nucl. Instrum. Methods Phys. Res., Sect. A 393, 129 (1997).

[18] J. P. Delahaye, G. Guignard, J. Irwin, T. O. Raubenheimer, R. D. Ruth, I. Wilson, and P. B. Wilson, in Proceedings of the 1997 Particle Accelerator Conference, Vancouver, Canada (IEEE, Piscataway, NJ, 1998), p. 482.

[19] H. P. Freund and P. G. O'Shea, Phys. Rev. Lett. 80, 520 (1998).

[20] D. X. Wang, G. A. Krafft, and C. K. Sinclair, Phys. Rev. E 57, 2283 (1998).

[21] G. A. Krafft, D. Wang, E. Price, and E. Feldl, in Proceedings of the 1995 Particle Accelerator Conference, Dallas, Texas (IEEE, Piscataway, NJ, 1996), p. 2601.

[22] S. Krinsky, M. L. Perlman, and R.E. Watson, in Handbook on Synchrotron Radiation, edited by E.E. Koch (North-Holland, Amsterdam, 1983), p. 152.
[23] R. P. Walker, Nucl. Instrum. Methods Phys. Res., Sect. A 267, 537 (1988).

[24] J.D. Jackson, Classical Electrodynamics (Wiley, New York, 1975), pp. 662-670.

[25] C. P. Neuman, M. L. Ponds, G. A. Barnett, J. M. J. Madey, and P. G. O'Shea, Nucl. Instrum. Methods Phys. Res., Sect. A 429, 287 (1999).

[26] O. V. Chubar, Rev. Sci. Instrum. 66, 1872 (1995).

[27] E. B. Blum, U. Happek, and A. J. Sievers, Nucl. Instrum. Methods Phys. Res., Sect. A 307, 568 (1991).

[28] W. S. Graves, E. D. Johnson, and T. O. Raubenheimer, in Proceedings of the XVIII International Linear Accelerator Conference, Geneva, 1996, edited by C. Hill and M. Vretenar (CERN, Geneva, 1996), p., 776.

[29] D. C. Quimby, S. C. Gottscahlk, F. E. James, K. E. Robinson, J. M. Slater, and A. S. Valla, Nucl. Instrum. Methods Phys. Res., Sect. A 285, 281 (1989). 